\title{
TRAP1 Inhibition Increases Glutamine Synthetase Activity in Glutamine Auxotrophic Non-small Cell Lung Cancer Cells
}

\author{
VU T.A. VO ${ }^{1,2}$, JONG-WHAN CHOI ${ }^{1}$, AI N.H. PHAN ${ }^{1,2}$, TUYEN N.M. HUA ${ }^{1,2}$, MIN-KYU KIM ${ }^{1,2}$, \\ BYOUNG HEON KANG ${ }^{5}$, SOON-HEE JUNG ${ }^{3}$, SUK-JOONG YONG ${ }^{4}$ and YANGSIK JEONG ${ }^{1,2}$ \\ ${ }^{1}$ Department of Biochemistry, ${ }^{2}$ Department of Global Medical Science, Medical Research Center, \\ Institute of Lifestyle Medicine, and Departments of ${ }^{3}$ Pathology and ${ }^{4}$ Internal Medicine, \\ Yonsei University Wonju College of Medicine, Wonju, Republic of Korea; \\ ${ }^{5}$ Department of Biological Sciences, Ulsan National Institute of Science and Technology (UNIST), \\ Ulsan, Republic of Korea
}

\begin{abstract}
Background/Aim: Cancer cells are distinct in terms of glutamine dependence. Here we investigated the different susceptibility of glutamine-independent and glutamine-dependent non-small cell lung cancer (NSCLC) to treatment with tumor necrosis factor receptorassociated protein 1 (TRAP1) inhibitor gamitrinibtriphenylphosphonium (G-TPP). Materials and Methods: Cell viability and proliferation under glutamine deprivation and G-TPP treatment were determined by the MTT and colony-formation assays. Protein and mRNA expression were determined by western blot and quantitative polymerase chain reaction. Colorimetric-based assay was performed to check for glutamine synthetase (GS) activity. Results: NSCLC cells showed diverse adaptation under glutamine-depleted condition and were categorized into glutamine-independent and glutamine-dependent cells. Treatment with G-TPP particularly increased GS activity and induced cell death due to energy shortage indicated by phosphorylated AMP-activated protein kinase (AMPK) in glutamine-dependent cells. Conclusion: This finding provides better understanding of TRAPl-mediated glutamine metabolism through GS activity, and evidence that TRAP1 could be a promising therapeutic target for glutamine-addicted cancer.
\end{abstract}

Correspondence to: Yangsik Jeong, Department of Biochemistry, Yonsei University Wonju College of Medicine, 20 Ilsan-ro, Wonju, Gangwon-do 26426, Republic of Korea. E-mail: yjeong@yonsei.ac.kr and Suk-Joong Yong, Department of Internal medicine, Yonsei University Wonju College of Medicine, 20 Ilsan-ro, Wonju, Gangwon-do 26426, Republic of Korea. E-mail: sjyong@yonsei.ac.kr

Key Words: Glutamine metabolism, TRAP1, gamitrinibtriphenylphosphonium, glutamine synthetase, lung cancer.
Cancer metabolism has become a hallmark described as reprogramming of nutrient utilization in cancer cells compared to that of their normal counterparts (1). The Warburg effect, or aerobic glycolysis, explains the higher glucose consumption of cancer cells and has become the central paradigm in the field of cancer metabolism. This biochemical principle practically accompanied the development of positron emission tomography-computed tomography into clinical application for imaging metabolically enhanced tumors, and following-up patients postoperatively for cancer relapse (2). Along with glucose as a preferable nutrient, the non-essential amino acid glutamine has become one of the most important nutrients, being involved in many biochemical pathways such as energy production, macromolecular synthesis, and scavenging under oxidative stress in cancer (3). Glutamine metabolism is dysregulated in various types of cancer which mostly display glutamine addiction for cell proliferation and thus show growth inhibition or cell death under glutamine-restricted conditions $(3,4)$. Two main cytosolic enzymes, glutaminase (GLS) and glutamine synthetase (GS), are involved in catabolic and anabolic processes of glutamine, respectively. GLS catalyzes glutamine to ammonium ion and glutamate which enters the tricarboxylic acid cycle to produce metabolic precursors for macromolecular synthesis or acts as a bioenergetic resource, while GS conversely generates glutamine from the reaction of coupling glutamate with ammonium ion in an ATP-dependent manner $(5,6)$. As a master regulator, oncogenic $c-M Y C$ transcriptionally regulates expression of subsets of genes involved in glutamine metabolism, in particular, of glutamine-addicted cancer cells (3). These include glutamine transporter solute carrier family 1 member 5 (SLC1A5) and glutamine catabolism enzymes such as $G L S(7,8)$. Interestingly, cMYC also induces GS expression in subsets of cancer cells for survival benefit under glutamine starvation (9). 
Particularly in a lung cancer model, c-MYCto was found to induce expression of both GLS and GS, which seems paradoxical since these two enzymes are functionally opposing (10). Notably, while GLS is well described as an important enzyme which leads to glutamine addiction, very few studies have investigated the role of GS in modulating glutamine utilization by cancer cells.

In addition to the reprograming of glucose and lipid metabolism $(11,12)$, NSCLC has been reported to be more dependent on glutamine for cell proliferation, compared to other cancer types (13). Here we focus on tumor necrosis factor receptor-associated protein 1 (TRAP1) function in regulating glutamine metabolism of NSCLC cells. TRAP1 is a downstream target of c-MYC (14) and a member of the mitochondrial heat-shock protein (HSP) 90 family functionally involved in protecting cells from DNA damage, apoptosis and other stress conditions $(15,16)$, which links TRAP1 overexpression to poor prognosis of patients with cancer (17). TRAP1 inhibition by gamitrinib-triphenylphosphonium (GTPP) led to a significant inhibition of cell viability and proliferation in cancer cells, including $\operatorname{NSCLC}(16,18,19)$. While the metabolic function of TRAP1 has been associated with glucose utilization and bioenergy synthesis (19-21), TRAP1 regulation of glutamine metabolism, especially through enzyme activity, remains unclear.

In the present study, exploiting inhibition by G-TPP, we investigated the function of TRAP1 in relation to GS in lung cancer cells.

\section{Materials and Methods}

Cell lines and reagents. Human NSCLC cell lines H1299, H2122, H358, H2073, H460, H2347, H1975, H1395 and H2085 were kindly provided by John D. Minna (University of Texas Southwestern Medical Center, Dallas, Texas, USA). H2122, H358, H2073, H460, H2347, H1975 cells were grown in RPMI 1640 (Corning, Mediatech Inc., Manassas, VA, USA) supplemented with $5 \%$ fetal bovine serum (FBS) (Gibco, Life Technologies Corporation, Grand Island, NY, USA), $50 \mathrm{U} / \mathrm{ml}$ penicillin and 50 $\mathrm{U} / \mathrm{ml}$ streptomycin (Gibco). H1395, H2085 cells were grown in RPMI 1640 (Corning) supplemented with 10\% FBS (Gibco), 50 $\mathrm{U} / \mathrm{ml}$ penicillin and $50 \mathrm{U} / \mathrm{ml}$ streptomycin (Gibco). H1299 was grown in Dulbecco's modified Eagle's medium (DMEM; Corning) supplemented with $5 \% \mathrm{FBS}, 50 \mathrm{U} / \mathrm{ml}$ penicillin and $50 \mathrm{U} / \mathrm{ml}$ streptomycin. The cells were maintained in a humidified incubator at $37^{\circ} \mathrm{C}$ with $5 \% \mathrm{CO} 2$. G-TPP was from LegoChem Biosciences (Daejeon, Republic of Korea).

Glutamine deprivation: media preparation. DMEM (D5030; Sigma, St. Louis, MO, USA) was reconstituted according to the supplier's instructions and $3.5 \mathrm{~g} / \mathrm{l} \mathrm{L-glucose} \mathrm{(Sigma)} \mathrm{added.} \mathrm{L-Glutamine}$ (Gibco) was omitted or added to a concentration of $2 \mathrm{mM}$ for full media.

Colony-formation assay. Cells were seeded in 6-well plates $\left(10^{4}\right.$ cells/well) in a total $2 \mathrm{ml}$ of culture media. The next day, cells were treated with 1, 2, 3, $5 \mu \mathrm{M}$ of G-TPP or dimethylsulfoxide (DMSO; Sigma) as control for 10 days. To visualize the colonies, cells were stained with methylene blue solution ( $0.4 \%$ in methanol $50 \%)$.

MTT assay. Cells were seeded in 96-well plates $\left(3 \times 10^{3}\right.$ cells/well) in a total $100 \mu \mathrm{l}$ of culture media. The next day, cells were treated with 1,2, 3, $5 \mu \mathrm{M}$ of G-TPP (or DMSO as control), or exposed to glutamine deprivation media (or full media as control) for 2 days. After treatment, cell viability was assayed using Thiazolyl Blue Tetrazolium Blue (MTT, $5 \mathrm{mg} / \mathrm{ml}$ in phosphate buffer saline; Sigma).

Western blot. $\mathrm{H} 358, \mathrm{H} 2122, \mathrm{H} 2073$, and $\mathrm{H} 1395$ cells cultured in full or glutamine deprivation media, or treated with $0,1,2,3,5 \mu \mathrm{M}$ of G-TPP, were obtained for western blot. Cell lysates were prepared using lysis buffer $(150 \mathrm{mM} \mathrm{NaCl}, 1 \%$ triton $\mathrm{X}-100,0.5 \%$ sodium deoxycholate, $0.1 \%$ sodium dodecyl sulfate (SDS), $50 \mathrm{mM}$ Tris $\mathrm{pH}$ 8) containing protease inhibitor and phosphatase inhibitor. Protein in cell lysates was measured by the BCA protein assay (Pierce Biotechnology, Rockford, IL, USA) and loaded in SDS polyacrylamide gel electrophoresis for western blot analysis. Primary antibody against TRAP1 from Abcam (Cambridge, MA, USA) was kindly provided by Professor Byoung Heon Kang. Primary antibodies against AMP-activated protein kinase (AMPK), phospho-AMPK (p-AMPK), acetyl-CoA carboxylase (ACC), pACC were from Cell Signaling Technology (Danvers, MA, USA), and that against $\beta$-actin was from Abcam. Quantification of western blot data was performed using ImageJ (22).

RNA isolation and quantitative polymerase chain reaction ( $q P C R)$. $\mathrm{H} 358, \mathrm{H} 2122, \mathrm{H} 2073$, and $\mathrm{H} 1395$ cell treated with $0,1,2 \mu \mathrm{M}$ of G-TPP were obtained for qPCR. Total RNA was prepared using TRIzol reagent (Invitrogen, Carlsbad, CA, USA) and used for cDNA synthesis by ReverTra Ace qPCR RT Master Mix (Toyobo, Osaka, Japan). qPCR was performed using an ABI Prism 7900 HT Sequence Detection System (Applied Biosystems, Foster City, CA, USA). Data were analyzed using the ddCt method with $18 S$ as internal control. Primers used for qPCR were: $18 \mathrm{~S}$ : forward 5'-ACCGCAGCTAGG AATGGA3', reverse 5'-GCCTCAGTTCCGAAAACC-3'; GS: forward 5'TGGGAGCAGACAGAGCCTAT-3', reverse 5'-AGGCATGACCT CGGCATTAG-3'; TRAP1: forward 5'-GACGCACCGCTCAACAT-3', reverse 5'-CACATCAAACATGGACGGTTT-3'.

Mitochondrial membrane potential measurement. Cells were seeded in black 96-well plates (Costar, Corning). The next day, cells were treated with G-TPP or DMSO as control. After $24 \mathrm{~h}$ of treatment, the medium was replaced by a solution of tetraethylbenzimidazolylcarbocianin iodide (JC-1; Invitrogen) in Krebs-Ringer Bicarbonate (KRB) and cells were incubated at $37^{\circ} \mathrm{C}$ with $5 \% \mathrm{CO}_{2}$ for 30 minutes. The $\mathrm{JC}-1$ solution was then replaced by KRB and fluorescence signals were read using FlexStation II (Molecular Devices, San Jose, CA, USA).

Assay of GS enzymatic activity. The method used was as described by Santoro et al. (23). In principle, GS has glutamyl transferase activity, catalyzing the reaction of glutamine and hydroxylamine to form $\gamma$-glutamyl hydroxamate, which is brown in the presence of ferric chloride. GS activity was measured based on the absorbance of this synthesized product $\gamma$-glutamyl hydroxamate. Briefly, cells were seeded at 100,000 cells per well in 12-well plates. The next day, cells were treated with 2, 3, $5 \mu \mathrm{M}$ of G-TPP or DMSO as control. After 24 hours, the medium was removed, and cells were 


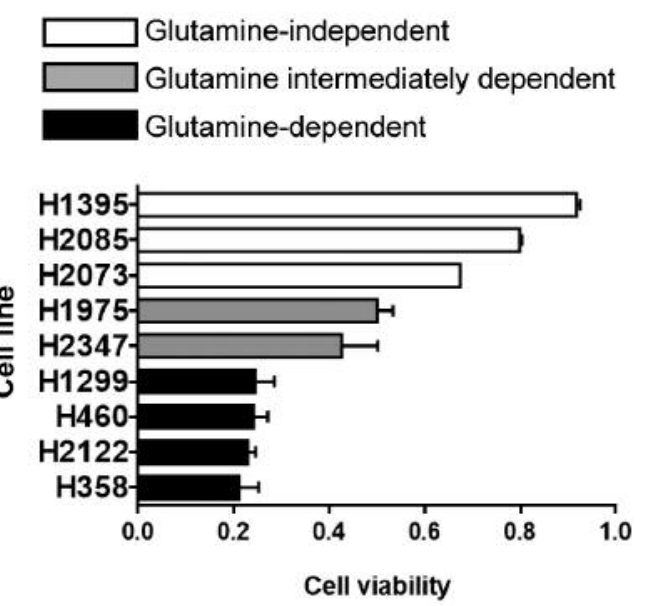

Figure 1. Non-small cell lung cancer (NSCLC) cell lines adapt differently to glutamine deprivation. Nine NSCLC cell lines were cultured in glutamine-depleted media for 48 hours, and cell viability was evaluated using MTT assay. Groups of cell lines were classified based on their glutamine dependence.

washed twice with phosphate buffer saline. Cells were then lysed by adding of $50 \mathrm{mM}$ imidazole $\mathrm{HCl} \mathrm{pH} 6.8$ to each well following one free-thaw cycle. One volume of assay buffer $(50 \mathrm{mM}$ imidazole, $25 \mathrm{mM}$ sodium arsenate, $50 \mathrm{mM}$ L-glutamine, $25 \mathrm{mM}$ hydroxylamine, $0.16 \mathrm{mM}$ adenosine diphosphate) was combined with cell lysate in each well. The plates were incubated at $37^{\circ} \mathrm{C}$ for $6 \mathrm{~h}$. At the end of the reaction, two volumes of stop buffer $(90 \mathrm{mM}$ ferric chloride in $1.8 \mathrm{~N} \mathrm{HCl}, 1.45 \%$ trichloroacetic acid) was added to each reaction well. The blank included $50 \mathrm{mM}$ imidazole $\mathrm{HCl} \mathrm{pH}$ 6.8 , assay buffer, and stop buffer (1:1:2). The mixture in each well was transferred to microtubes, centrifuged and the absorbance of the supernatant at $540 \mathrm{~nm}$ was read using a spectrophotometer (Versamax, Molecular Devices).

Statistical analysis. Student's $t$-test was used to compare means of two data groups. One-way ANOVA was used to compare means of two or more data groups.

All graphs and statistical analysis were prepared using GraphPad Prism version 7.0 (GraphPad Softwave, La Jolla, CA, USA). Data are reported as the mean $\pm S D(n \geq 3)$, and significance was accepted at $p \leq 0.05$.

\section{Results}

NSCLC cell lines show divergent adaptation to glutamine deprivation. We first explored the relative glutamine dependency of nine NSCLC cell lines, namely H1395, H2085, H2073, H1975, H2347, H1299, H460, H2122, and H358. Cell viability assay showed neither necessarily lethality nor resistance of all cell lines, but a range of sensitivity to glutamine deprivation which was arbitrarily further classified into three groups (Figure 1). The first group consisting of glutamine-independent cell lines, showing viability higher than $60 \%$ when cultured for 48 hours under glutamine-free conditions, includes H1395, H2085 and H2073 cells. Secondly, H1975 and H2347 cell lines comprise an intermediately responding group with $40-60 \%$ sustained cell viability. Lastly, four cell lines, H1299, H460, H2122 and H358, showing high glutamine dependency with viability remaining under $30 \%$ without glutamine, comprised glutamine-dependent cell lines. We then focused on the separate glutamine-dependent and -independent groups for further experiments.

Glutamine-addicted NSCLC cell lines are highly sensitive to TRAP1 inhibitor. Since TRAP1, a downstream target of oncogenic c-MYC, plays a critical role in glucose metabolism but little is known of its role in glutamine metabolism (19-21), here we wondered if the glutamine auxotrophic feature is associated with TRAP1 function. To investigate TRAP1 function in glutamine metabolism, we first examined protein expression of both $\mathrm{c}-\mathrm{MYC}$ and TRAP1 in the two groups of NSCLC lines. We found that glutamine-dependent cell lines showed significantly higher expression of c-MYC compared to glutamine-independent cells (Figure 2A). This observation is consistent with previous studies on c-MYC function as a master regulator leading to glutamine addiction in cancer cells $(7,10)$. TRAP1 protein, however, showed a somewhat diverse expression pattern between the two groups (Figure 2A). This suggests that TRAP1 expression level may not necessarily be correlated with glutamine dependency. When treated with TRAP1 inhibitor G-TPP (Figure 2B), interestingly, glutamine-dependent cell lines H2122 and H358 showed remarkable sensitivity to dose-dependent growth inhibition, but glutamine-independent ones did not (Figure 2C).

It might be suggested that the growth-inhibitory effect of G-TPP is due to a reduction of mitochondrial membrane potential as reported previously (18); however, we found no significant changes of the membrane potential in H2122 and H1395 cells, representing glutamine-dependent and independent cell lines, respectively, upon G-TPP treatment (Figure 2D). This result excludes the possibility that the distinct growth response of glutamine-dependent cells to G-TPP is due to changes in mitochondrial membrane potential.

Differently from other cancer types in which c-MYC regulates glutamine metabolism mostly through GLS, in lung cancer particularly, c-MYC may also be involved in expression and activity of GS (10). We next focused on the relationship of TRAP1 and GS, expecting that higher expression of c-MYC in glutamine-dependent cell lines may lead to up-regulation of GS expression. We found that GS is indeed up-regulated in glutamine-dependent H2122 and H358 cells compared to glutamine-independent H2073 and H1395 cells (Figure 2E, left). However, G-TPP treatment did not alter the mRNA levels of TRAPl or GS in glutamine- 


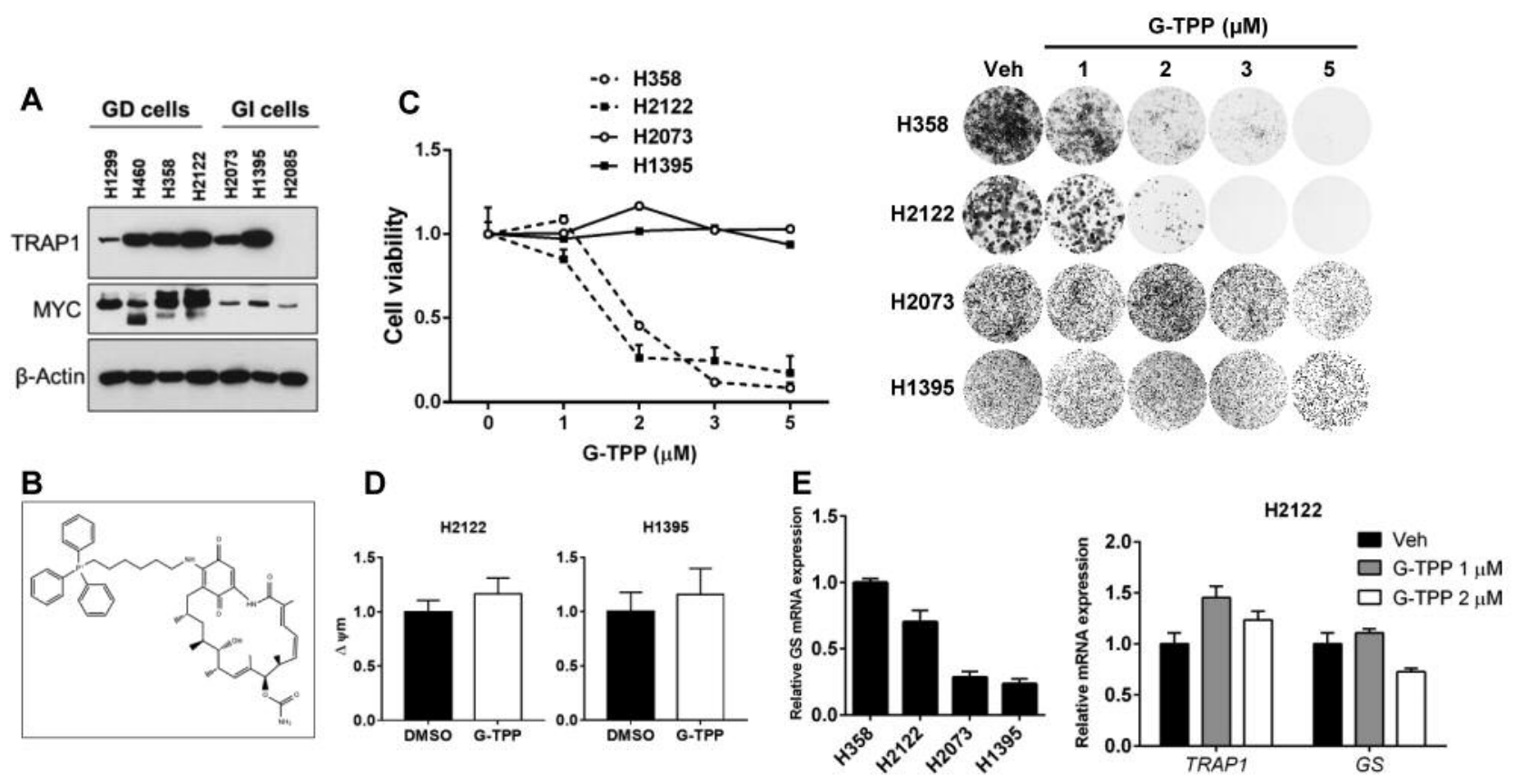

Figure 2. Non-small cell lung cancer (NSCLC) cell lines are selectively sensitive to tumor necrosis factor receptor-associated protein 1 (TRAP1) inhibitor gamitrinib-triphenylphosphonium $(G-T P P)$ independently of TRAP1 expression. A: TRAP1 and MYC protein expression in glutaminedependent $(G D)$ and -independent (GI) cell lines. B: Chemical structure of G-TPP. C: G-TPP-mediated growth inhibition of lung cancer cell lines. Cell viability was assayed upon G-TPP treatment using MTT (left) and liquid colony-formation (right) assays. D: Measurement of mitochondrial membrane potential in glutamine-dependent $H 2122$ and glutamine-independent H1395 lung cancer cells. Cells were treated with $2 \mu M$ of G-TPP for $24 \mathrm{~h}$ and followed by JC-1 assay to monitor the polarization of mitochondrial membrane. E: mRNA expression of glutamine synthetase (GS) and TRAP1. Basal levels of GS mRNA expression were measured using quantitative polymerase chain reaction in both glutamine-dependent (H358 and H2122) and glutamine-independent (H2073 and H1395) cell lines (left). mRNA expression of TRAP1 and GS was measured using qPCR in H2122 cells upon G-TPP treatment (right).Veh: Vehicle.

dependent H2122 cells (Figure 2E, right). These observations may indicate a potential mechanism by which TRAP1 inhibition regulates glutamine metabolism, particularly in glutamine-dependent cell lines, regardless of TRAP1 and GS expression. Hence, we next investigated TRAP1 inhibitionmediated GS enzymatic activity.

G-TPP treatment releases GS enzymatic activity in glutamine-addicted NSCLC cells. As not clearly associated with TRAP1 and GS protein expression, we further investigated the effect of TRAP1 regulation on GS activity. As briefly shown in Figure 3A, GS enzymatic activity was assayed by measuring the product of GS catalytic activity, $\gamma$-glutamyl hydroxamate. Glutamine-dependent H358 and H2122 cells showed higher basal GS activity compared to the glutamine-independent H2073 and H1395 cells (Figure 3B). Next, both groups of cells were treated with TRAP1 inhibitor G-TPP in a dose-dependent manner followed by measurement of GS activity. Surprisingly, glutaminedependent cells, but not -independent cells, showed strong increase of GS activity with G-TPP treatment in a dose- dependent manner (Figure 3C and D). This suggests that pharmacological inhibition of TRAP1 may unblock GS activity in cells which are addicted to glutamine even they show lower basal GS activity, which might result in the different growth-inhibitory effects of TRAP1 inhibitor between two cell lines groups linked to glutamine metabolism.

G-TPP treatment increases phosphorylation of AMPK and thus its downstream target acetyl co-A carboxylase in glutamine-dependent NSCLC cells. As GS activity is critical for suppressing glutamine-dependent cell lines, we rationalized that increasing GS activity by G-TPP treatment may increase the intracellular glutamine level, thus reducing glutamate entering the TCA cycle, which may result in reduction of ATP production (5). In this aspect, we investigated if increase of GS activity by TRAP1 inhibition increases phosphorylation of AMPK, a hallmark for reduction of ATP generation. To that end, we first examined the effects of glutamine on the phosphorylation of AMPK in both groups. Consistently, without glutamine supply, glutamine- 
A

Glutamine + hydroxylamine

Glutamine synthetase

(GS)

v-Glutamyl hydroxamate

Ferric chloride

Spectrophotometric measurement at $540 \mathrm{~nm}$
B

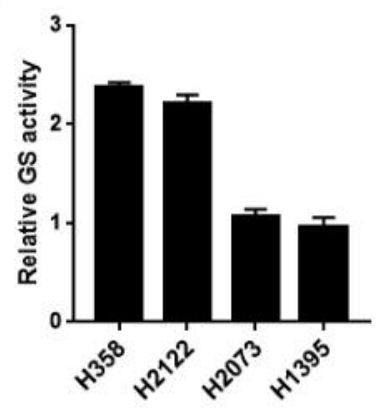

C

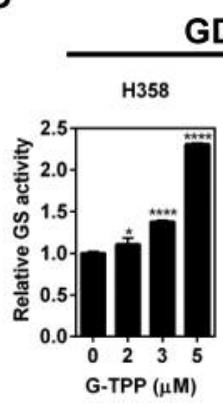

GD cells

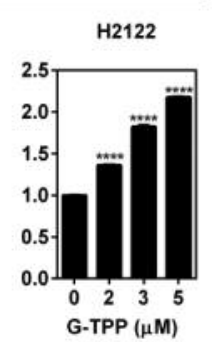

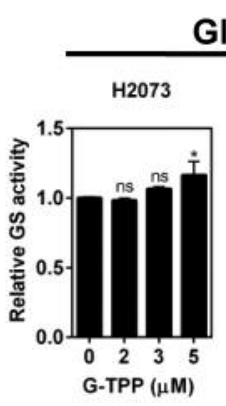

GI cells

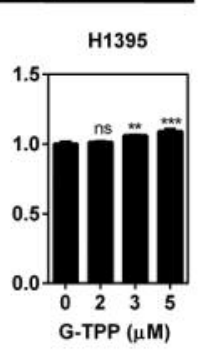

D

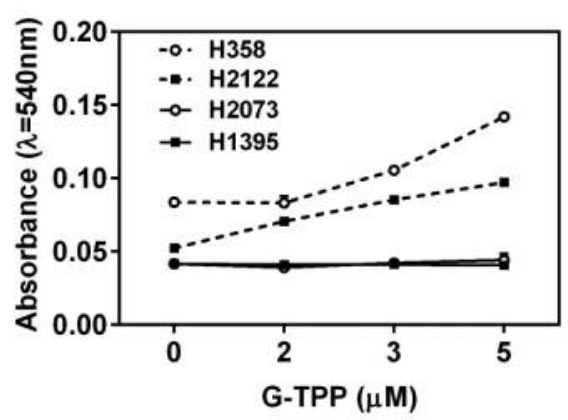

Figure 3. Gamitrinib-triphenylphosphonium (G-TPP)-mediated tumor necrosis factor receptor-associated protein 1 (TRAP1) inhibition releases glutamine synthetase (GS) enzymatic activity. A: Brief principle of GS enzymatic activity. Basal GS activity (B) in glutamine-dependent (GD) (H358 and H2122) and glutamine-independent (GI) (H2073 and H1395) cell lines and upon G-TPP treatment (C) was measured and data were normalized with cell viability. D: Increase in absorbance at $540 \mathrm{~nm}$ due to $\gamma$-glutamyl hydroxamate production on treatment with G-TPP. Significantly different from the untreated control at $* p \leq 0.05, * * p \leq 0.01, * * * p \leq 0.001, * * * * p \leq 0.0 .0001 . n s, p>0.05$.
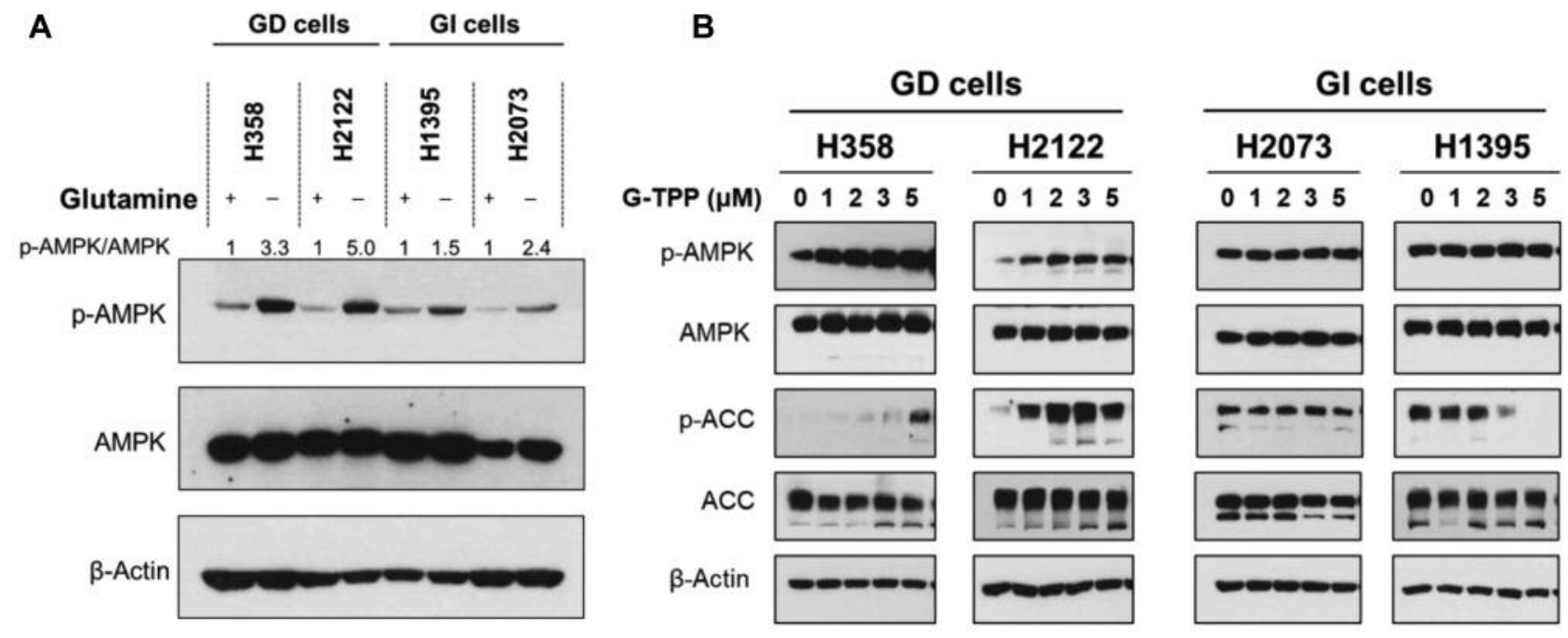

Figure 4. Tumor necrosis factor receptor-associated protein 1 (TRAP1) inhibition increases AMP-activated protein kinase (AMPK) activity. A: Glutamine-dependent (GD) (H358 and H2122) and glutamine-independent (GI) (H2073 and H1395) cell lines were cultured in glutamine-depleted media for $24 \mathrm{~h}$ and analyzed by western blot for expression of phospho-AMPK ( $p$-AMPK). B: Cells were treated with G-TPP in a dose-dependent manner and analysed by western blot for expression of $p$-AMPK and phospho-acetyl-CoA carboxylase (p-ACC). 
independent cell lines, which may not require glutamine for ATP production, showed a lesser increase of p-AMPK than did the glutamine-dependent cell lines (Figure 4A). Furthermore, treatment with TRAP1 inhibitor G-TPP significantly induced p-AMPK and thus phosphorylation of acetyl-coA carboxylase in glutamine-dependent cells while in glutamine-independent cells it did not (Figure 4B). These results suggest the susceptibility of glutamine-dependent cells to glutamine deprivation may be due to reduced bioenergetics.

\section{Discussion}

Glutamine, the most abundant amino acid in the blood, serves as a source of carbon and nitrogen for biosynthesis, bioenergetics and maintenance of redox balance in many cancer types. This so-called "preferred nutrient" is a necessary supplement for the in vitro growth of a subsets of cancer cells termed glutamine auxotrophs, in which oncogenic c-MYC plays a critical role in transcription regulation of target genes involved in glutamine and glucose metabolism (3). Here, in order to elucidate the molecular and biochemical mechanism for glutamine dependency in glutamine auxotrophs, we utilized groups of NSCLC cell lines, glutamine-dependent and glutamine-independent, based on their susceptibility to cell death under glutamine deprivation.

Consistent with previous reports $(7,8,24)$, we confirmed c-MYC up-regulation in cell lines which are deeply dependent on glutamine for cell growth. Moreover, we also found enzymatic activity of GS to be higher in the glutamine-dependent cell lines, which is seemingly paradoxical since it increases the intracellular level of glutamine produced from glutamate. Thus, we further rationalized that glutamine-addicted cell lines might exploit glutamine predominantly as a carbon source for energy production through anaplerotic process $(25,26)$, in addition to acting as a nitrogen source for biosynthesis and reducing potential for redox maintenance. High GS activity may reduce the level of glutamate entering the TCA cycle and thus reduce ATP generation, which eventually leads to cell death. In this rationale, we proposed a hypothesis that TRAP1, as a downstream player of c-MYC, increases ATP generation from glutamine-derived sources by suppressing GS activity.

As a pharmacological approach to inhibiting TRAP1, we utilized G-TPP, a TRAP1 inhibitor developed to specifically target the HSP90 network in the mitochondrial matrix (18) and thus potentiate its anticancer effect. Several preclinical studies revealed that G-TPP alone, or in combination with doxorubicin, had favorable anticancer effects and safety in various types of cancer, including glioma and prostate cancer $(27,28)$. In NSCLC, which has been reported to acquire drug resistance to targeted therapy such as tyrosine kinase inhibitors (29), further efforts are needed to overcome this challenge (30). Encouragingly, G-TPP treatment was found to reduce tumor volume in a xenograft model and defeated gefitinib resistance by inducing apoptosis $(18,31)$. Likewise, in the present study, we found that treatment with TRAP1 inhibitor G-TPP released GS activity and suppressed cell growth only in the glutamine-dependent NSCLC cell lines. Knowing that TRAP1 inhibition modulates AMPK as an indicator of ATP reduction in several cancer cell types including lung cancer $(32,33)$, we also found an increase of p-AMPK upon G-TPP treatment, which only occurred in glutamine-dependent NSCLC cells. Taken together, these data show that TRAP1 inhibition releases GS activity and may reduce the intracellular level of glutamate entering the TCA cycle, which affects only the cells with biased utilization of glutamine for ATP production. Since GS expression and activity has been shown to be up-regulated by c-MYC (9), TRAP1 may be considered as a feedback regulator modulating GS activity and thereby glutamine metabolism. This study provides a mechanistic insight into TRAP1 inhibition of GS activity for therapeutically targeting glutamine auxotrophic cancer.

\section{Acknowledgements}

This work was financially supported by the Basic Science Research Program through the National Research Foundation of Korea funded by the Ministry of Education, Science and Technology (NRF2016R1D1A3B03930581 to YS Jeong), Medical Research Center Program (2017R1A5A2015369), and a grant of the Korea Health Technology R\&D Project through the Korea Health Industry Development Institute (KHIDI), funded by the Ministry of Health \& Welfare, Republic of Korea (HI17C0039).

\section{References}

1 DeBerardinis RJ and Chandel NS: Fundamentals of cancer metabolism. Sci Adv 2: e1600200, 2016.

2 Liberti MV and Locasale JW: The Warburg effect: how does it benefit cancer cells? Trends Biochem Sci 41: 211-218, 2016.

3 Altman BJ, Stine ZE, and Dang CV: From Krebs to clinic: glutamine metabolism to cancer therapy. Nat Rev Cancer 16: 619-634, 2016.

4 van den Heuvel AP, Jing J, Wooster RF and Bachman KE: Analysis of glutamine dependency in non-small cell lung cancer: GLS1 splice variant GAC is essential for cancer cell growth. Cancer Biol Ther 13: 1185-1194, 2012.

5 Newsholme P, Procopio J, Lima MM, Pithon-Curi TC and Curi $\mathrm{R}$ : Glutamine and glutamate - their central role in cell metabolism and function. Cell Biochem Funct 21: 1-9, 2003.

6 Chen L and Cui H: Targeting glutamine induces apoptosis: a cancer therapy approach. Int J Mol Sci 16: 22830-22855, 2015.

7 Wise DR, DeBerardinis RJ, Mancuso A, Sayed N, Zhang XY, Pfeiffer HK, Nissim I, Daikhin E, Yudkoff M, McMahon SB and Thompson CB: MYC regulates a transcriptional program that stimulates mitochondrial glutaminolysis and leads to glutamine addiction. Proc Natl Acad Sci USA 105: 18782-18787, 2008. 
8 Shajahan-Haq AN, Cook KL, Schwartz-Roberts JL, Eltayeb AE, Demas DM, Warri AM, Facey CO, Hilakivi-Clarke LA and Clarke R: MYC regulates the unfolded protein response and glucose and glutamine uptake in endocrine resistant breast cancer. Mol Cancer 13: 239, 2014.

9 Bott AJ, Peng IC, Fan Y, Faubert B, Zhao L, Li J, Neidler S, Sun Y, Jaber N, Krokowski D, Lu W, Pan JA, Powers S, Rabinowitz J, Hatzoglou M, Murphy DJ, Jones R, Wu S, Girnun G and Zong WX: Oncogenic Myc Induces Expression of Glutamine Synthetase through Promoter Demethylation. Cell Metab 22: 1068-1077, 2015.

10 Yuneva MO, Fan TW, Allen TD, Higashi RM, Ferraris DV, Tsukamoto T, Mates JM, Alonso FJ, Wang C, Seo Y, Chen X and Bishop JM: The metabolic profile of tumors depends on both the responsible genetic lesion and tissue type. Cell Metab 15: 157170, 2012

11 Killock D: Metabolic heterogeneity and paradoxical glucose usage in NSCLC. Nat Rev Clin Oncol 13: 202, 2016.

12 Phan ANH, Vo VTA, Hua TNM, Kim MK, Jo SY, Choi JW, Kim HW, Son J, Suh YA and Jeong Y: PPARgamma sumoylationmediated lipid accumulation in lung cancer. Oncotarget 8 : 82491-82505, 2017.

13 Lee JS, Kang JH, Lee SH, Lee CH, Son J and Kim SY: Glutaminase 1 inhibition reduces thymidine synthesis in NSCLC. Biochem Biophys Res Commun 477: 374-382, 2016.

14 Coller HA, Grandori C, Tamayo P, Colbert T, Lander ES, Eisenman RN and Golub TR: Expression analysis with oligonucleotide microarrays reveals that MYC regulates genes involved in growth, cell cycle, signaling, and adhesion. Proc Natl Acad Sci USA 97: 3260-3265, 2000.

15 Altieri DC, Stein GS, Lian JB and Languino LR: TRAP-1, the mitochondrial Hsp90. Biochim Biophys Acta 1823: 767-773, 2012

16 Rasola A, Neckers L and Picard D: Mitochondrial oxidative phosphorylation TRAP(1)ped in tumor cells. Trends Cell Biol 24: 455-463, 2014.

17 Agorreta J, Hu J, Liu D, Delia D, Turley H, Ferguson DJ, Iborra F, Pajares MJ, Larrayoz M, Zudaire I, Pio R, Montuenga LM, Harris AL, Gatter K and Pezzella F: TRAP1 regulates proliferation, mitochondrial function, and has prognostic significance in NSCLC. Mol Cancer Res 12: 660-669, 2014.

18 Kang BH, Plescia J, Song HY, Meli M, Colombo G, Beebe K, Scroggins B, Neckers L and Altieri DC: Combinatorial drug design targeting multiple cancer signaling networks controlled by mitochondrial HSP90. J Clin Invest 119: 454-464, 2009.

19 Sciacovelli M, Guzzo G, Morello V, Frezza C, Zheng L, Nannini $\mathrm{N}$, Calabrese F, Laudiero $\mathrm{G}$, Esposito $\mathrm{F}$, Landriscina $\mathrm{M}$, Defilippi P, Bernardi P and Rasola A: The mitochondrial chaperone TRAP1 promotes neoplastic growth by inhibiting succinate dehydrogenase. Cell Metab 17: 988-999, 2013.

20 Chae YC, Angelin A, Lisanti S, Kossenkov AV, Speicher KD, Wang H, Powers JF, Tischler AS, Pacak K, Fliedner S, Michalek RD, Karoly ED, Wallace DC, Languino LR, Speicher DW and Altieri DC: Landscape of the mitochondrial HSP90 metabolome in tumours. Nat Commun 4: 2139, 2013.

21 Lisanti S, Tavecchio M, Chae YC, Liu Q, Brice AK, Thakur ML, Languino LR and Altieri DC: Deletion of the mitochondrial chaperone TRAP-1 uncovers global reprogramming of metabolic networks. Cell Rep 8: 671-677, 2014.
22 Schneider CA, Rasband WS and Eliceiri KW: NIH Image to ImageJ: 25 years of image analysis. Nat Methods 9: 671-675, 2012.

23 Santoro JC, Harris G and Sitlani A: Colorimetric detection of glutamine synthetase-catalyzed transferase activity in glucocorticoid-treated skeletal muscle cells. Anal Biochem 289: 18-25, 2001.

24 Qing G, Li B, Vu A, Skuli N, Walton ZE, Liu X, Mayes PA, Wise DR, Thompson CB, Maris JM, Hogarty MD and Simon MC: ATF4 regulates MYC-mediated neuroblastoma cell death upon glutamine deprivation. Cancer Cell 22: 631-644, 2012.

25 DeBerardinis RJ, Mancuso A, Daikhin E, Nissim I, Yudkoff M, Wehrli $\mathrm{S}$ and Thompson CB: Beyond aerobic glycolysis: transformed cells can engage in glutamine metabolism that exceeds the requirement for protein and nucleotide synthesis. Proc Natl Acad Sci USA 104: 19345-19350, 2007.

26 Fan J, Kamphorst JJ, Mathew R, Chung MK, White E, Shlomi T and Rabinowitz JD: Glutamine-driven oxidative phosphorylation is a major ATP source in transformed mammalian cells in both normoxia and hypoxia. Mol Syst Biol 9: 712, 2013.

27 Kang BH, Siegelin MD, Plescia J, Raskett CM, Garlick DS, Dohi T, Lian JB, Stein GS, Languino LR and Altieri DC: Preclinical characterization of mitochondria-targeted small molecule HSP90 inhibitors, gamitrinibs, in advanced prostate cancer. Clin Cancer Res 16: 4779-4788, 2010.

28 Park HK, Lee JE, Lim J, Jo DE, Park SA, Suh PG and Kang $\mathrm{BH}$ : Combination treatment with doxorubicin and gamitrinib synergistically augments anticancer activity through enhanced activation of Bim. BMC Cancer 14: 431, 2014.

29 Workman P and Clarke PA: Resisting targeted therapy: fifty ways to leave your EGFR. Cancer Cell 19: 437-440, 2011.

30 Phan AN, Hua TN, Kim MK, Vo VT, Choi JW, Kim HW, Rho JK, Kim KW and Jeong Y: Gallic acid inhibition of SRC-STAT3 signaling overcomes acquired resistance to EGF receptor tyrosine kinase inhibitors in advanced non-small cell lung cancer. Oncotarget 7: 54702-54713, 2016.

$31 \mathrm{Kim} \mathrm{H}$, Oh S, Jo J, Hwang K and Jeong E: Inhibitory effects of mitochondrial TRAP1 on gefitinib-resistance in non-small lung cancer cells. Respirology 22: 111-111, 2017.

32 Caino MC, Chae YC, Vaira V, Ferrero S, Nosotti M, Martin NM, Weeraratna A, O'Connell M, Jernigan D, Fatatis A, Languino LR, Bosari S and Altieri DC: Metabolic stress regulates cytoskeletal dynamics and metastasis of cancer cells. J Clin Invest 123: 2907-2920, 2013.

33 Chae YC, Caino MC, Lisanti S, Ghosh JC, Dohi T, Danial NN, Villanueva J, Ferrero S, Vaira V, Santambrogio L, Bosari S, Languino LR, Herlyn M and Altieri DC: Control of tumor bioenergetics and survival stress signaling by mitochondrial HSP90s. Cancer Cell 22: 331-344, 2012.
Received January 31, 2018

Revised February 25, 2018

Accepted February 27, 2018 